\title{
Endosomal trafficking of open Major Histocompatibility Class I conformers-Implications for presentation of endocytosed antigens ${ }^{\text {is }}$
}

\author{
Hana Mahmutefendića ${ }^{\mathrm{a}}$ Gordana Blagojević Zagorac ${ }^{\mathrm{a}}$, Maja Ilić Tomaša ${ }^{\mathrm{a}}$, Marcus Groettrup ${ }^{\mathrm{b}}$, \\ Frank Momburg ${ }^{\mathrm{c}}$, Pero Lučin ${ }^{\mathrm{a}, *}$ \\ a University of Rijeka, Faculty of Medicine, Department of Physiology and Immunology, B. Branchetta 20, 51000 Rijeka, Croatia \\ b University of Konstanz, Department of Biology/Immunology, Postbox M661, Universitaetsstr. 10, D-78457 Konstanz, Germany \\ ${ }^{\mathrm{c}}$ Division of Translational Immunology, German Cancer Research Center and NCT, Im Neuenheimer Feld 280, 69120 Heidelberg, Germany
}

\begin{abstract}
A B S T R A C T
Major Histocompatibility Class I (MHC-I) molecules are present at the cell surface either as fully conformed trimolecular complexes composed of heavy chain, beta-2-microglobulin $(\beta 2 \mathrm{~m})$ and antigenic peptide or as various open forms, devoid of the peptide and/or $\beta 2 \mathrm{~m}$. While the role of fully conformed MHC-I is well studied, the physiological role of open conformers is neglected. We have shown that fully conformed MHC-I and open MHC-I conformers segregate at the PM and during endosomal trafficking resulting in the exclusion of open MHC-I from the early endosomal/juxtanuclear recycling route. As a result, open MHC-I conformers are internalized with a higher rate than fully conformed counterparts. Although the majority of internalized open MHC-I is directed into the acidic late endosomal (LE) compartments, only a fraction of them is degraded. Namely, a significant fraction of open MHC-I is present in a subset of LEs with the capacity of recycling and/or exocytosis. Therefore, it should be examined whether exogenous peptide loading may occur during traveling of MHC-I proteins through LE compartments, especially in a subset of less acidic LEs that detach from the core of perinuclear acidic LEs and migrate toward the cell periphery. Given that the acidic LE environment is not favorable for peptide loading, an endosomal compartment with the recycling capacity and less acidic environment that allows stabilization of newly formed trimolecular complexes is proper site for exogenous peptide loading. We propose that a LE compartment which collect and retain open MHC-I conformers should be taken into consideration as a site of exogenous peptide loading.
\end{abstract}

\section{Introduction}

Major Histocompatibility Class I (MHC-I) molecules are present at the cell surface and in the intracellular membraneous compartments as fully conformed trimolecular complexes composed of heavy chain, peptide and $\beta-2$ microglobulin (full MHC-I) and as open MHC-I conformers (heavy chain without or with very weakly bound $\beta-2$ microglobulin). A majority of open MHC-I conformers localize inside the cell in membraneous compartment that belong to the endosomal system. After more than two decades of MHC-I

Abbreviations: $\mathrm{EE}$, early endosome; $\mathrm{eL}^{\mathrm{d}}$, empty $\mathrm{L}^{\mathrm{d}}$; $\mathrm{fL}^{\mathrm{d}}$, full $\mathrm{L}^{\mathrm{d}}$; $\mathrm{IR}$, internalization rate; MHC-I, Major Histocompatibility Class I; PM, plasma membrane; LE, late endosome; TEE, tubular early endosomes; TfR, transferrin receptor; VEE, vacuolar early endosomes.

is This article belongs to Special Issue on Antigen Processing and Presentation.

* Corresponding author at: Department of Physiology and Immunology, University of Rijeka, Faculty of Medicine, Braće Branchetta 20, 51000 Rijeka, Croatia. Tel.: +385 51406 500; fax: +385 51675699 .

E-mail addresses: pero.lucin@uniri.hr, pero@uniri.hr (P. Lučin). research, physiological implications of open MHC-I conformers are still poorly understood and their existence at the cell surface and in the endosomal system is not properly validated. Still, it is not known how and where cell surface and endosomal open MHC-I conformers are generated, especially in the light of well established endoplasmic reticulum (ER) quality control machinery that recognize empty MHC-I as unfolded and either retrieves them in the ER until they become peptide loaded or redirect them into the cytosol for proteasomal degradation (reviewed by Donaldson and Williams, 2009). It is, thus, an open question whether similar quality control machinery operates at the cell surface and in the endosomal system. Furthermore, it is not clear how they behave in the endosomal system, what are sorting principles that drive their endosomal trafficking and what are implications for process of alternative antigen presentation. Therefore, physiological importance of open MHC-I conformers should be clarified not only for peptide loading in the endosomal system and regulation of the immune response but also for understanding of protein sorting in the endosomal hemisphere of intracellular membranous organelles. Here, we will describe our current understanding of 
endosomal trafficking of open MHC-I conformers and discuss their potential role in presentation of endocytosed antigen.

\section{Current status}

Open MHC-I conformers of murine and human classical MHC-I alleles are present at the cell surface of various cell lines (Zagorac et al., 2012). Their expression is $2-5$ times lower than expression of fully conformed MHC-I counterparts and increases with the overall increase of MHC-I expression, i.e. after IFN-gamma treatment or after aberrant expression by transfection. In some cells, the amount of open MHC-I conformers at the cell surface may override the amount of fully conformed MHC-I after IFN-gamma treatment.

Surface MHC-I proteins are internalized by the constitutive endocytic uptake of the plasma membrane (PM) (Donaldson and Williams, 2009) which is variable in different cells, ranging from $50 \%$ to $180 \%$ membrane equivalent every hour (Huotari and Helenius, 2011). Internalization rate (IR) of membrane proteins, i.e. their loss from the cell surface, is resultant of their endocytic rate (rate of translocation of a PM protein into the cell interior by endocytic uptake) and the recycling rate (rate of return of endocytosed PM protein from cell interior back to the PM). Thus, proteins that do not recycle will have the same endocytic and internalization rate. Fully conformed MHC-I proteins internalize with the rate $0.002-0.004 \mathrm{~min}^{-1}(0.2-0.4 \%$ loss of initially surface expressed molecules per minute, i.e. $12-24 \%$ per hour) which is $5-8$-fold lower than IR of their open forms $\left(0.011-0.022 \mathrm{~min}^{-1}\right)$, resulting in their 5-8 times longer half-life at the cell surface (Zagorac et al., 2012). This difference in the IRs suggests segregation within membranes, distinct endocytic route and distinct recycling efficiency of MHC-I conformers.

Fully conformed MHC-I proteins and open MHC-I conformers segregate already at the PM by partitioning into distinct membrane environment (Fig. 1A): fully conformed MHC-I molecules localize in the lipid-disordered membrane composition whereas open MHC-I conformers partition into the lipid-organized membrane environment (Mahmutefendić et al., 2011; Zagorac et al., 2012). Thus, it can be expected that their constitutive endocytic uptake engages different endocytic machinery, although both fully conformed MHC-I and open MHC-I are constitutively endocytosed by a mechanism that do not require clathrin and dynamin (Donaldson and Williams, 2009). This is supported by the observation that they are not captured into the same endocytic invaginations and primary endocytic carriers (Mahmutefendić et al., 2011). Upon endocytosis, primary endocytic carriers loaded with fully conformed MHC-I and open MHC-I conformers converge into same early endosomes but maintain the preference for distinct membrane composition and partition into distinct membrane domains (Mahmutefendić et al., 2011; Zagorac et al., 2012).

In early endosomes (EEs), complex organelles with tubular and vacuolar domains (Huotari and Helenius, 2011), MHC-I conformers continue to segregate. Approximately one-third of endocytosed fully conformed MHC-I proteins segregate into tubular EEs (TEEs), accessing early endosomal recycling route together with transferrin receptor (TfR), whereas all open MHC-I conformers remain in vacuolar domain of EEs (VEEs) together with the rest of fully conformed MHC-I (Fig. 1A). With progression of VEEs toward the cell center, roughly a half of fully conformed MHC-I proteins that remained in VEEs continue to segregate into tubular extensions, which localize in the juxtanuclear area and collect almost all endocytosed TfR but not open MHC-I conformers. These endosomes are known as juxtanuclear recycling compartment (JRC). The rest of fully conformed MHC-I proteins remains together with open MHC-I conformers in EEA1-positive VEEs (Fig. 1A), localize in the perinuclear area, grow in size with time and mature into late endosomes (Mahmutefendić et al., 2011). Thus, a substantial fraction of endocytosed fully conformed MHC-I proteins recycle via TEEs and tubular JRC, whereas no open MHC-I conformers were detected to recycle via this route (Mahmutefendić et al., 2011; Zagorac et al., 2012). Although fully conformed MHC-I recycle with the similar rate as TfR, this rate cannot explain their IR in relation with the more rapid constitutive membrane uptake. In addition, constitutive membrane uptake is also higher then IR of open MHC-I conformers. Thus, rapid recycling route of both fully conformed MHC-I and open MHC-I should be considered and examined. Both fully conformed MHC-I and open MHC-I internalize with much higher rate when recycling is inhibited by aluminum fluoride (Zagorac et al., 2012), which inhibits recycling in the entire endosomal system, including rapid recycling.

As previously explained, endocytosed open MHC-I conformers are sorted in the perinuclear region into vacuolar EEs that enlarge, mature into acidic LEs (Fig. 1A) and acquire major LE markers Lamp1 and Lamp2. Although it is believed that MHC-I proteins which reach LE compartments are predestined for degradation, our recent studies in fibroblastic cell lines showed that endocytosed open MHC-I conformers are segregated and retained in LE compartment outside the bulk of acidic perinuclear LEs (Fig. 1A). Given that these endosomes maintain their multivesicular and vacuolar nature, we propose that this compartment represents a LE subset with recycling capacity (Huotari and Helenius, 2011). As a result, the internal LEs vesicles will be shed out into the extracellular environment as exosomes, while the limiting membrane of LE will become integral part of the PM which will results in recycling of associated cargo proteins (Fig. 1A). Therefore, the destiny of molecules that have reached LEs could be multiple: retention, degradation, exocytosis, or recycling. In line with this, we observed that acidic and Lamp $1^{\text {high }}$ perinuclear LEs are accessed by internalized EGF-EGFR, cargo destined for degradation, and that $50-60 \%$ of internalized open $\mathrm{L}^{\mathrm{d}}$ is degraded. The rest of internalized open $\mathrm{L}^{\mathrm{d}}$ is redirected into less acidic and Lamp $1^{\text {low }}$ vacuolar endosomes outside the perinuclear area at the cell periphery and very often in extensions of fibroblasts (Mahmutefendić et al., manuscript in preparation). These, peripheral Lamp $1^{\text {low }}$ LEs are accessed by fluid phase cargo (dextran), internalized open MHC-I conformers, and are free of degradation cargo (EGF-EGFR). Accordingly, we have found that $10-15 \%$ of internalized open $\mathrm{L}^{\mathrm{d}}$ is recycled via LE route (Mahmutefendić et al., manuscript in preparation).

Acidic endosomal compartments play an important role in alternative antigen presentation. It has been shown that endosomal acidification is necessary for processing of long antigens (membrane associated or not) as a source for alternative antigen presentation. This endosomal processing pathway functions not only in TAP-deficient but also in wild type nonprofessional APCs (Tiwari et al., 2007).

For understanding of mechanisms leading to cross-presentation and alternative presentation pathways involving "recycling" endosomes it is essential to fully understand the endocytic trafficking of MHC-I proteins, their behavior in the acidic environment of endosomes and consider the role of open MHC-I conformers. In order to test for sensitivity of MHC-I proteins in endosomal environment we examined the ratio of full and open MHC-I molecules (murine $\mathrm{H} 2-\mathrm{L}^{\mathrm{d}}$ allele) at the cell surface exposed to various acidic conditions for 30 min (Fig. 1B and C). As shown in Fig. 1B, very small amount of $\mathrm{fL}^{\mathrm{d}}$ is converted into $\mathrm{eL}^{\mathrm{d}}$ when the $\mathrm{pH}$ is maintained above 6.0 ( $\mathrm{pH}$ of EEs and recycling endosomes), whereas reduction of $\mathrm{pH}$ below 6.0 (which occurs in LEs) facilitated conversion of $\mathrm{fL}^{\mathrm{d}}$ into $\mathrm{eL}^{\mathrm{d}}$. Prolonged exposure to $\mathrm{pH} 6.0$ also converted $\mathrm{fL}^{\mathrm{d}}$ into $\mathrm{eL}^{\mathrm{d}}$ (Fig. 1C). Similar was observed also for human MHC-I alleles (data not shown). Therefore, if fully conformed MHC-I proteins are exposed to $\mathrm{pH} 6.0$ for $30 \mathrm{~min}$, very little of them will lose the peptide and became empty, ready to acquire free peptide. Fully conformed 
A

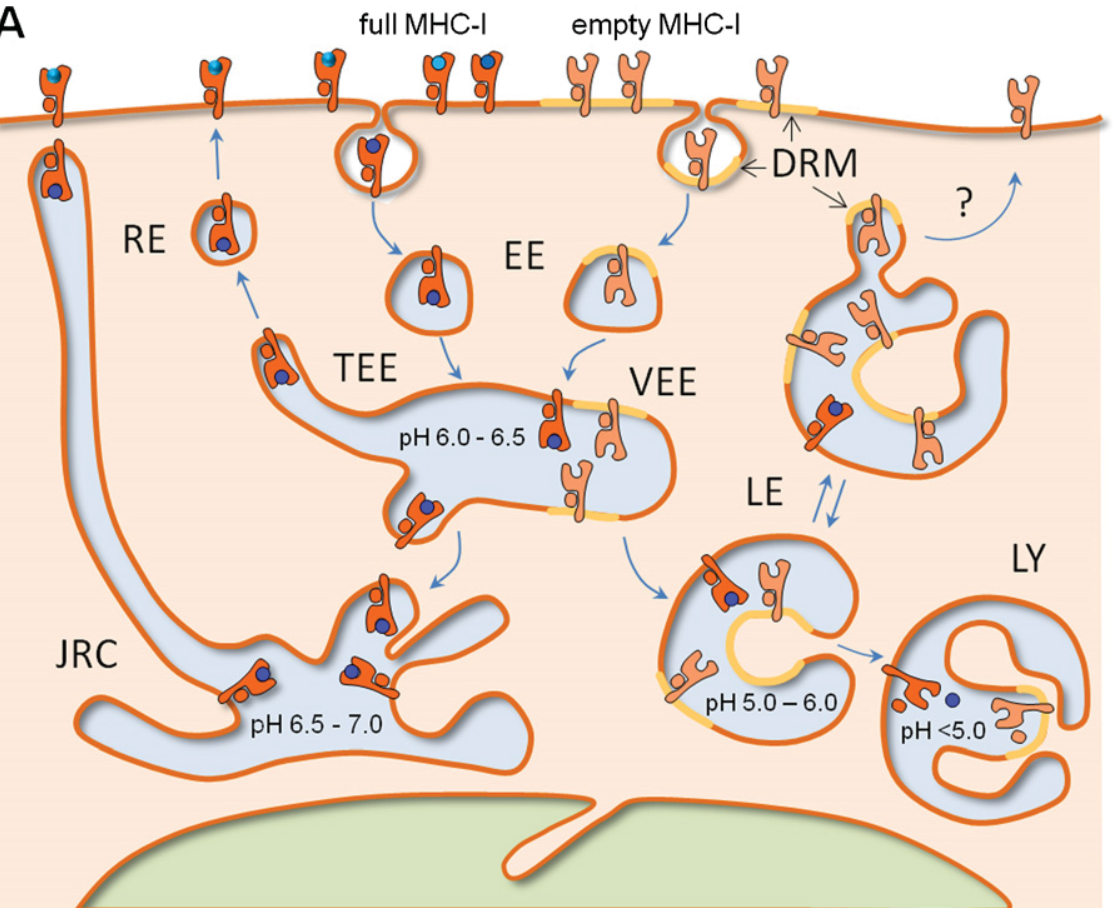

B

C
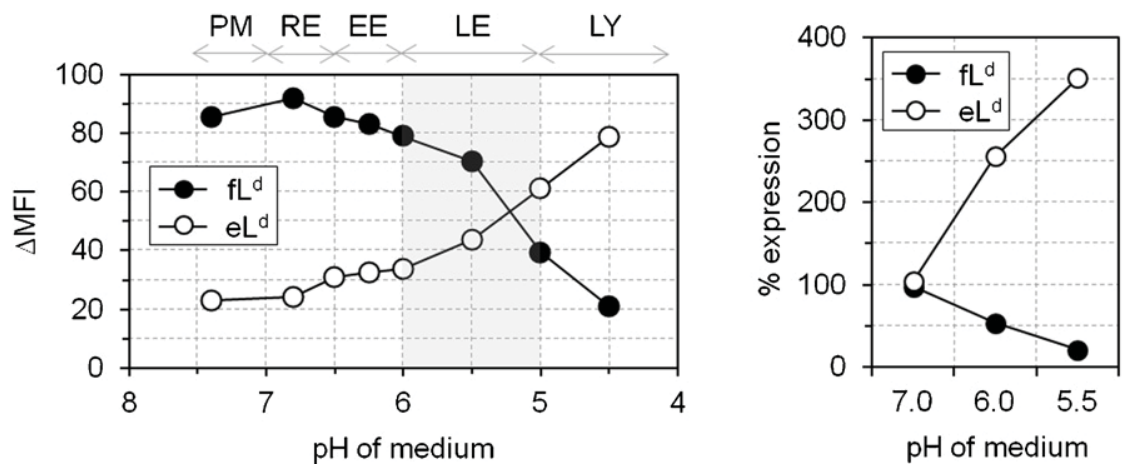

Fig. 1. Endocytic routes and segregation of MHC-I proteins in the endosomal system. (A) Schematic representation of endosomal trafficking of fully conformed MHC-I proteins (loaded peptide is represented by blue circles) and open MHC-I conformers (without peptide). (B) Cell surface expression of fully conformed $\mathrm{H} 2$ - $\mathrm{L}^{\mathrm{d}}$ ( $\mathrm{fL}^{\mathrm{d}}$ ) and peptideempty H2- $\mathrm{L}^{\mathrm{d}}$ conformers $\left(\mathrm{eL}^{\mathrm{d}}\right)$ after 30 min exposure of P815 cells to various pH environments. Data represent mean fluorescence intensity (determined by flow cytometry) after staining with primary antibodies (30-5-7 and 64-3-7, respectively) and FITC-conjugated secondary antibody subtracted for fluorescence intensity of cells exposed to secondary reagent only $(\triangle \mathrm{MFI})$. (C) Cell surface expression of $\mathrm{fL}^{\mathrm{d}}$ and $\mathrm{eL}^{\mathrm{d}}$ after $2 \mathrm{~h}$ exposure of P815 cell to various pH environments. EE, early endosomes; TEE, tubular early endosomes; VEE, vacuolar early endosomes; RE, recycling endosomes; JRC, juxtanuclear recycling compartment; LE, late endosomes; LY, lysosomes; DRM, detergent resistance membranes.

MHC-I proteins, remain in EEs for 15-20 min and, therefore, one cannot expect their conversion into empty form and loading with a free peptide. Similarly, it is unlikely that conversion and peptide loading can occur in recycling endosomes where $\mathrm{pH}$ is even higher. Thus, the preferred options would be peptide exchange, either with peptide that is of a higher affinity and endocytosed in a high concentration into EE or simultaneous trafficking of peptide and MHC-I into LE from where MHC-I molecules with exchanged peptide will recycle to the cell surface.

\section{Future perspectives}

Understanding of endosomal sorting of fully conformed MHC-I proteins and open MHC-I conformers has several important implications in understanding protein sorting in the endosomal system, organization of the quality control in the endosomal system, function and organization of late endosomal compartments, biogenesis and physiology of MHC-I proteins and, consequently, the understanding of peptide loading in the vacuolar pathway.

Proper understanding of the peptide loading in the endosomal system will require identification of the endosomal compartment where peptide loading and exchange can occur. Such a compartment (i) should be able to retain MHC-I proteins, especially open MHC-I conformers, (ii) should be able to acquire peptides either endocytosed by fluid phase uptake or by proteolytic degradation in acidic endosomes, (iii) should be able to exchange cargo with acidic LEs and protect peptides from extensive degradation, (iv) should be able to reduce acidity in order to allow proper folding of loaded MHC-I proteins, and (v) should be able to return loaded MHC-I proteins back to the cell surface. Thus, it is possible that alternative peptide loading takes place during traveling of MHC-I molecules through classical acid LEs and a subsequent sorting into subset of less acidic LEs that are targeted to the PM. The acidic environment of 
LEs would partially disrupt the conformation of MHC-I molecules, especially along peptide binding grove, thereby allowing the new peptide to be bound. Finally, the less acidic pH in the "recycling" LEs would allow stabilization of those newly formed trimolecular MHC-I complexes.

A major understanding of cross-presentation came from studies on dendritic cells and macrophages which are key players in the initiating of the immune response in the lymph nodes. However, very little is known about the role of open MHC-I conformers in these cells. Thus studies performed in fibroblasts, mastocytoma cells and carcinoma cell lines regarding endosomal MHC-I trafficking and sorting (Mahmutefendić et al., 2011; Zagorac et al., 2012) should be confirmed on professional antigen presenting cells isolated ex vivo.

\section{References}

Donaldson, J.G., Williams, D.B., 2009. Intracellular assembly and trafficking of MHC class I molecules. Traffic 10, 1745-1752.

Huotari, J., Helenius, A., 2011. Endosomal maturation. EMBO Journal 30, 3481-3500. Mahmutefendić, H., Blagojević, G., Ilić Tomaš, M., Kučić, N., Lučin, P., 2011. Segregation of open Major Histocompatibility class I conformers at the plasma membrane and during endosomal trafficking reveals conformation-based sorting in the endosomal system. International Journal of Biochemistry and Cell Biology 43, 504-515.

Tiwari, N., Garbi, N., Reinheckel, T., Moldenhauer, G., Hämmerling, G.J., Momburg, F., 2007. A transporter associated with antigen-processing independent vacuolar pathway for the MHC class I-mediated presentation of endogenous transmembrane proteins. Journal of Immunology 178, 7932-7942.

Zagorac, G.B., Mahmutefendić, H., Ilić Tomaš, M., Kučić, N., Le Bouteiller, P., Lučin, P. 2012. Early endosomal rerouting of Major Histocompatibility class I conformers. Journal of Cellular Physiology 227, 2953-2964. 